\title{
A plethora of human pluripotent stem cells
}

\begin{abstract}
At the early stages of mammalian development, a number of developmentally plastic cells appear that possess the ability to give rise to all of the differentiated cell types normally derived from the three primary germ layers - unique character known as pluripotency. To date, embryonic stem cells (ESCs) and induced pluripotent stem cells (iPSCs) have been shown to be truly pluripotent. However, recent studies have revealed a variety of other cells that demonstrate pluripotentiality, including very small embryonic-like stem cells (VSELs), amniotic fluid stem cells (AFSCs), marrow-isolated adult multilineage inducible cells (MIAMI) and multipotent adult precursor cells (MAPCs). This review summarises key features of these six kinds of pluripotent and potentially pluripotent stem cells (ESCs, iPSCs, VSELs, AFSCs, MIAMI and MAPCs) and the evidence for their pluripotency properties.
\end{abstract}

Keyword: Amniotic fluid stem cells; Induced pluripotent stem cells; MAPCs; MIAMI;

Pluripotent stem cells; Very small embryonic-like stem cells 